\title{
O uso da kinesio taping no tratamento da paralisia facial pós-acidente vascular cerebral fase aguda
}

\author{
The use of kinesio taping in the treatment of the acute phase of \\ post-stroke facial paralysis
}

\author{
Simone Rosa Barreto ${ }^{1}$ (1) Aline Mansueto Mourão ${ }^{2}$ (1) Tatiana Simões Chaves ${ }^{3}$ (D) Laelia Cristina Caseiro Vicente ${ }^{2}$ (D)
}

\begin{abstract}
RESUMO
Objetivo: comparar os resultados da reabilitação da paralisia facial pósacidente vascular cerebral isquêmico na fase aguda, com e sem o uso da Kinesio Taping. Métodos: estudo experimental caso e controle com 46 pacientes com paralisia facial pós-acidente vascular cerebral, distribuídos em dois grupos de forma randomizada, para a reabilitação da mímica facial: o grupo caso realizou terapia miofuncional orofacial e fez uso da Kinesio Taping nos músculos zigomáticos maior e menor e o grupo controle apenas terapia miofuncional orofacial. Para avaliação da paralisia facial, foi utilizada a escala de House e Brackmann e o protocolo de incompetência do movimento para as medições da face. Todos os participantes realizaram 12 dias de intervenção fonoaudiológica para a reabilitação da mímica facial. Para análise, considerou-se a incompetência do movimento por meio das medidas da face e o grau de comprometimento da paralisia facial e foi verificado se a idade poderia ter influenciado os resultados. Foram realizadas análises de associação e o nível de significância adotado foi de $5 \%$. Resultados: os dois grupos apresentaram melhora da assimetria facial após intervenção fonoaudiológica, a incompetência do movimento foi menor em todas as medidas da face e a melhora da gravidade da paralisia facial foi semelhante, sem diferença estatística entre os tratamentos. Conclusão: tanto a terapia miofuncional orofacial exclusiva, como associada ao uso da Kinesio Taping, são estratégias terapêuticas que promovem melhora da paralisia facial pós-acidente vascular cerebral.
\end{abstract}

Palavras-chave: Paralisia facial; Kinesio; Acidente Vascular Cerebral; Reabilitação; Fonoaudiologia

\begin{abstract}
Purpose: To compare the results of treatment of facial paralysis after ischemic stroke in the acute phase with and without the use of Kinesio Taping. Methods: Experimental case-control study with 46 patients with facial paralysis after stroke, randomly assigned to two groups for treatment of facial mimicry: the case group underwent orofacial myofunctional therapy and used Kinesio Taping on the zygomatic major and minor muscles while the control group only received orofacial myofunctional therapy. To assess facial paralysis, the House and Brackmann scale and the movement incompetence protocol were used for facial measurements. All participants underwent 12 days of treatment for facial mimicry. The analysis considered movement incompetence by means of the face measurements and the degree of impairment of facial paralysis, checking whether age might have influenced the results. Association analyses were performed and the significance level adopted was 5\%. Results: both groups showed an improvement in facial asymmetry after treatment, movement incompetence was lower in all facial measurements, and the improvement in the severity of facial paralysis was similar, with no statistical difference between treatments. Conclusion: Both the exclusive orofacial myofunctional therapy and the one combined with Kinesio Taping are therapeutic strategies that promote improvement in post-stroke facial paralysis.
\end{abstract}

Keywords: Facial paralysis; Kinesio; Stroke; Rehabilitation; Speech Therapy

\footnotetext{
Trabalho realizado no Hospital Risoleta Tolentino Neves, Universidade Federal de Minas Gerais - UFMG - Belo Horizonte (MG), Brasil.

${ }^{1}$ Programa de Residência Integrada Multiprofissional em Saúde, Ênfase em Saúde do Idoso, Hospital Risoleta Tolentino Neves - HRTN - Belo Horizonte (MG), Brasil.

${ }^{2}$ Departamento de Fonoaudiologia, Faculdade de Medicina, Universidade Federal de Minas Gerais - UFMG - Belo Horizonte (MG), Brasil.

${ }^{3}$ Unidade de Acidente Vascular Cerebral, Hospital Risoleta Tolentino - HRTN - Belo Horizonte (MG), Brasil.

Conflito de interesses: Não.

Contribuição dos autores: SRB foi responsável pela coleta de dados, revisão de literatura e redação do artigo; TSC foi responsável pela análise dos resultados e revisão do artigo; AMM foi responsável pela redação, orientação do estudo e revisão do artigo; LCCV foi responsável pela concepção e delineamento do estudo, orientação do estudo, redação, revisão do artigo e aprovação final da versão a ser publicada.
}

Financiamento: Nada a declarar.

Autor correspondente: Simone Rosa Barreto. E-mail: simonerbarreto09@gmail.com

Recebido: Janeiro 11, 2021; Aceito: Junho 03, 2021 


\section{INTRODUÇÃO}

A paralisia facial (PF) é uma sequela comum em pacientes que tiveram acidente vascular cerebral (AVC) $)^{(1-4)}$. Estudo identificou que quase a metade dos indivíduos pós-AVC pesquisados apresentou $\mathrm{PF}^{(1)}$. Tal sequela ocorre devido à interrupção da informação motora para os músculos da face, por alteração na via motora facial central e por lesões dos neurônios motores piramidais do córtex frontal que chegam aos núcleos motores do facial ipsilateralmente e contralateralmente ${ }^{(3-7)}$.

Na paralisia facial central (PFC), ocorre o acometimento da mobilidade e simetria facial dos músculos do terço inferior da face contralateral à lesão, podendo estar associadas a outros sintomas neurológicos, como disartria, apraxias, disfagias, alterações motoras e/ou déficit de linguagem ${ }^{(2,5-7)}$.

A literatura aponta que os indivíduos com paralisia dos músculos da face, além das alterações na mímica facial, mastigação, deglutição e fala, apresentam, também, problemas psicológicos e sociais, pois os movimentos da expressão facial garantem a efetividade da comunicação entre os indivíduos, importante no processo de socialização ${ }^{(8-10)}$.

A bandagem elástica Kinesio Taping (KT) foi criada em 1973 pelo Dr. Kenzo Kase. Para fixar na pele, a KT dispõe de uma cola especial sem látex, que permite a respiração cutânea sem obstruções e não possui medicamentos em sua composição ${ }^{7-12}$. É um recurso externo flexível para auxílio no corpo humano, que pode estirar-se, mas retorna ao seu tamanho original, permitindo, assim, a movimentação da musculatura em que foi aplicado $^{(8-13)}$. A fita KT atua estimulando e ativando os músculos durante os movimentos, melhorando a contração do músculo enfraquecido, inibido ou hipotônico, reduzindo episódios de fadiga, contraturas, espasmos e lesões musculares ${ }^{(13-15)}$.

Na Fonoaudiologia, a KT ainda é utilizada de forma empírica, uma vez que as pesquisas científicas se iniciaram recentemente ${ }^{(16)}$. Possui como princípios a analgesia, a adequação do tônus e melhora da função muscular ${ }^{(17-20)}$. Estudos indicaram o seu benefício no controle do escape extraoral de saliva, visto que melhorou a propriocepção local e o fortalecimento do músculo orbicular da boca ${ }^{(16,17)}$. Nas disfunções temporomandibulares, promoveu analgesia, relaxamento muscular, melhorando a circulação sanguínea e linfática na região próxima da articulação temporomandibular (ATM $)^{(18)}$. Na reabilitação das disfonias, a KT reduziu a tensão nos músculos cervicais ${ }^{(19)} \mathrm{e}$, na deglutição, melhorou a propriocepção e o fortalecimento dos músculos orofaciais $^{(20)}$.

Novos recursos terapêuticos estão sendo utilizados, de forma complementar à terapia fonoaudiológica das PFs, com objetivo de otimizar o tempo de tratamento. Tais recursos incluem eletroestimulação, fotobiomodulação, ou bandagem elástica associada à terapia miofuncional orofacial ${ }^{(10-13)}$.

No caso da reabilitação dos pacientes pós-AVC, a hipótese deste estudo é que a KT favorece a reorganização dos neurônios do nervo facial a partir de estímulo sensorial, ocorrendo a neuroplasticidade neuronal.

O sistema nervoso ( $\mathrm{SN}$ ) é composto por neurônios sensoriais, motores e de associação. Os receptores sensoriais aferem as informações ao sistema nervoso central (SNC), onde serão integradas por neurônios de associação, ou interneurônios, que enviam uma resposta eferente ao músculo. Assim, o SN detecta estímulos externos e internos, tanto físicos quanto químicos, e desencadeia as respostas musculares ${ }^{(16-19)}$. As áreas corticais possuem a capacidade de mudar, de se adaptar e de moldar-se em nível estrutural e funcional, fenômeno que acontece em decorrência desses estímulos ${ }^{(19,20)}$. Com base nesse princípio, a KT seria um estímulo externo físico que pode promover a neuroplasticidade do nervo facial no SNC por meio da estimulação tegumentar dos mecanorreceptores da pele, causando alteração do comportamento das unidades motoras dos músculos e excitação neuronal, ou seja, a bandagem colada na pele permite estímulos constantes, que são percebidos em nível cortical, e produzem resposta motora no sistema tegumentar, auxiliando na neuroplasticidade do sistema nervoso central ${ }^{(19,21)}$.

Por se tratar de um recurso ainda muito recente na prática clínica fonoaudiológica, a literatura é escassa em estudos que padronizam a utilização da bandagem elástica. Sendo assim, este estudo visou comparar os resultados da terapia miofuncional orofacial exclusiva e associada ao uso da KT na reabilitação da paralisia facial pós-AVC na fase aguda.

\section{MÉTODOS}

Trata-se de um estudo experimental caso e controle, realizado na Unidade de Acidente Vascular Cerebral - AVC do Hospital Risotela Tolentino Neves, com os pacientes internados no período de outubro de 2017 a agosto de 2018. O estudo foi aprovado pelo Comitê de Ética em Pesquisa da Universidade Federal de Minas Gerais, sob o parecer $n^{\circ}$ 3.082.696. Todos os participantes do estudo assinaram o Termo de Consentimento Livre e Esclarecido, concordando com os termos da pesquisa.

Foi realizada busca ativa nos prontuários eletrônicos, por meio do CID 10 - I64 (Classificação Internacional de Doenças 10 -I64 - acidente vascular cerebral não especificado como isquêmico ou hemorrágico, para verificar o perfil dos pacientes internados. Foram incluídos aqueles com diagnóstico de AVC na fase aguda (em até 72 horas de ictus) e paralisia facial, idade superior a 18 anos, nível de consciência satisfatório, de acordo com a escala de Glasgow (pontuação > 13) e linguagem preservada (Language Screening Test - LAST adequado) ${ }^{(21)}$, além de apresentarem a pele da face íntegra, sem cicatrizes, verrugas ou feridas e ausência de contraindicação médica para os procedimentos realizados no presente estudo. Foram excluídos pacientes com outras deformidades craniofaciais, doenças degenerativas, apraxia, diagnóstico de câncer em região de cabeça e pescoço, história prévia de $\mathrm{PF}$, intubados, traqueostomizados, pacientes grávidas, pacientes com paralisias faciais não decorrentes do AVC e aqueles que, por algum motivo, não completaram ou não realizaram todos os procedimentos empregados neste estudo.

Para seleção dos participantes, foram coletados os dados nos prontuários sobre laudo neurológico, resultados da tomografia computadorizada (TC), locais e extensão da lesão no encéfalo, gravidade do comprometimento neurológico, de acordo com a National Institute of Health Stroke Scale (NIHSS) ${ }^{(2)}$, deficit funcional, por meio da Medida de Independência Funcional $(\mathrm{MIF})^{(23)}$, e alteração miofuncional orofacial mediante protocolo de avaliação fonoaudiológica do serviço. Além disso, foi verificada com a equipe multiprofissional a possibilidade de alguma contraindicação aos procedimentos realizados.

Para avaliação da paralisia facial, foi utilizado o protocolo de classificação de gravidade House e Brackmann para $\mathrm{PF}^{(3,24-25)}$ e o protocolo de incompetência do movimento, incompetência do movimento $=$ lado paralisado - lado normal x 100/lado normal (IM=LP-LN x 100/LN) para as medições da face ${ }^{(26)}$. A escala de House e Brackmann foi adaptada para avaliação dos pacientes com PFC: em repouso, foi avaliada a simetria 
facial, analisando os três terços da face e, em movimento, foi considerado apenas o terço inferior de face ${ }^{(7)}$. Embora a escala avalie os três terços da face, vale mencionar que ela apresentou boa concordância intra-avaliadores e interavaliadores ${ }^{(25)}$, sendo possível utilizá-la para avaliar o terço inferior da face e, por esse motivo, considerou-se adequada a sua utilização na avaliação das PFC.

Os músculos analisados foram: occipitofrontal, corrugador do supercílio, piramidal/transverso do nariz, levantador do lábio superior e da asa do nariz, depressor do septo nasal, orbicular dos olhos, levantador do lábio superior, zigomático maior e menor, risório, abaixador do ângulo da boca, mentoniano e orbicular dos lábios. Os movimentos faciais solicitados foram "cara de assustado", "cara de bravo", "cara de cheiro ruim", "fechar os olhos suavemente", "fechar os olhos com força", "sorriso aberto", "sorriso fechado", "cara de triste", "beiço" e "bico". Os pacientes foram fotografados sentados no leito, com iluminação natural do ambiente e distância de, aproximadamente, 70 centímetros, com a câmera do celular Iphone 7 de 12 MP, gravação de vídeo 2160 p e resolução 750 x 1334 px.

Durante o procedimento de medição da face, os participantes mantiveram o olhar fixo à frente, uma vez que os olhares para cima e laterais poderiam alterar os valores obtidos; foi exigido esforço máximo para os movimentos solicitados durante a medição. Todos os procedimentos de avaliação da mímica facial foram realizados no mesmo dia e sempre pela mesma avaliadora.

Participaram do estudo 46 sujeitos com PF, constituídos em dois grupos de 23 pacientes cada um, alocados de forma randomizada; no grupo caso, a reabilitação foi composta por terapia miofuncional orofacial e utilização da KT nos músculos zigomáticos maior e menor e, no grupo controle, apenas a terapia miofuncional orofacial. Embora nos sorrisos com os lábios abertos e fechados outros músculos contraiam, no movimento, por questões metodológicas, optou-se por aplicar a KT apenas nos músculos zigomáticos maior e menor, devido à maior ação deles no movimento do sorriso aberto, para evitar a sobreposição de fitas e a interferência da bandagem nas funções orofaciais.

Para verificar alergia à KT, foi aplicado um pedaço de, aproximadamente, cinco centímetros de fita no braço dos pacientes do grupo caso, por um período de 24 horas. Após resposta negativa, foi realizada a aplicação da Kinesio Taping, em tiras com formato de I, tensão de $25 \%$ na zona terapêutica, mais precisamente no ventre dos músculos zigomáticos, com a ancoragem inicial em superfície malar do osso zigomático e ancoragem final em ângulo da boca e lábio superior, durante o período de internação do paciente (Figura 1). O tamanho da tira de bandagem foi definido individualmente, de acordo com

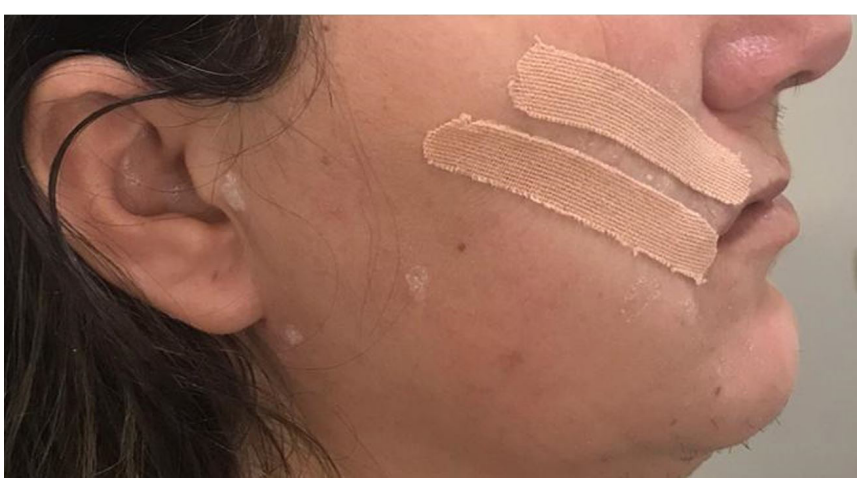

Figura 1. Foto de aplicação da fita de Kinésio nos músculos zigomáticos maior e menor a anatomia palpatória dos músculos, considerando a ancoragem na origem e inserção deles. Cada participante permaneceu três dias consecutivos com a bandagem e, após o terceiro dia, ficou sem o uso da fita por 24 horas para descanso da pele. Depois do período de repouso, uma nova tira da KT foi colocada na face do paciente pela fonoaudióloga responsável, permanecendo por mais três dias, repetindo esse procedimento até o indivíduo completar 12 dias de intervenção.

Ambos os grupos realizaram o protocolo de intervenção fonoaudiológica de terapia miofuncional orofacial do tipo manobras indutoras do movimento, com três sessões de dez repetições dos movimentos faciais alterados, três vezes ao dia ${ }^{(25,26)}$. Pela manhã, a intervenção foi realizada pela terapeuta e nos outros períodos (tarde e noite) pelo paciente e/ou acompanhante, que foram instruídos quanto à realização de cada tarefa com orientações verbais e escritas. Os pacientes do grupo caso foram orientados a realizar as massagens por cima da fita Kinesio Tape.

Para as manobras indutoras do movimento, todos os pacientes foram treinados a contrair e manter a contração dos músculos em ambos os lados da face, enquanto eram realizadas as massagens lentas e profundas no sentido da contração muscular, apenas no lado paralisado ${ }^{(27)}$. Quando os músculos começaram a apresentar movimento na fase de reinervação da $\mathrm{PF}$, foram iniciados os exercícios miofuncionais isotônicos e isométricos com os músculos da mímica facial, sendo os seguintes os exercícios isotônicos: contrair e soltar rapidamente o nariz, alternar rapidamente bico e sorriso fechado, alternar rapidamente bico e sorriso aberto, todos com dez repetições. Os exercícios isométricos foram: contrair o nariz, bico com os lábios fechados e depois abertos, sorriso com os lábios fechados e depois abertos, mantendo os movimentos por cinco segundos com cinco repetições ${ }^{(27)}$. As tarefas propostas deveriam ser realizadas com auxílio do feedback visual (espelho) ${ }^{(6)}$.

Após três dias de intervenção, tanto o grupo caso, quanto o grupo controle foram submetidos à reavaliação da mímica facial por meio dos mesmos protocolos usados na avaliação ${ }^{(25-27)}$. No total, todos os indivíduos que participaram do estudo realizaram 12 dias de intervenção fonoaudiológica para a reabilitação da mímica facial. Para análise dos dados, consideraram-se como variáveis respostas a incompetência do movimento por meio das medidas da face e o grau de comprometimento da paralisia facial e foi verificado se a idade poderia ter influenciado os resultados.

Para a análise descritiva, foi realizada a distribuição de frequência de todas as variáveis categóricas e análise das medidas de tendência central e de dispersão das variáveis contínuas. Para a análise de associação, foi utilizado o teste t de Student para amostras pareadas, além dos testes Qui-Quadrado de Pearson, Exato de Fisher, Wilcoxon e regressão ordinal simples. O nível de significância adotado em todas as análises foi de 5\%. O programa IBM SPSS Statistics versão 24.0 foi utilizado para processamento e análise dos dados.

\section{RESULTADOS}

A maioria dos pacientes era de idosos $(52,17 \%)$, do sexo masculino (60,87\%), com idade variando de 44 a 89 anos, média de 62,4 anos (desvio padrão $\pm 11,7$ anos), comprometimento neurológico moderado na escala NIHSS e dependência modificada com assistência em até 50\% nas tarefas na escala MIF nos dois grupos. A maioria dos indivíduos $(93,48 \%)$ apresentou paralisia facial central à esquerda $(63,4 \%)$. Na escala de gravidade, no 
grupo caso prevaleceu a PF moderada $(43,48 \%)$, seguida da moderada grave $(21,74 \%)$ e, no grupo controle, $\mathrm{PF}$ moderada (34,78\%), seguida da grave $(26,09 \%)$ e da leve (26,09\%). O tempo médio entre o ictus e o início da intervenção fonoaudiológica foi de 2,96 dias (desvio padrão $\pm 2,64$ dias). Apenas a variável sexo obteve significância estatística, sendo o masculino maior no grupo controle e o feminino maior no grupo caso; já nas demais variáveis os grupos foram iguais (Tabela 1).

Avaliando as medidas de face dos dois grupos, foi possível perceber que houve diferença significativa em quase todas as comparações entre a pré e a pós-intervenção. Em todos os casos, a média das medidas foi maior na pré-intervenção e, apesar de algumas medidas não alcançarem significância estatística, suas médias também foram maiores na pré-intervenção. No entanto, pelo teste, elas foram consideradas semelhantes. Observou-se, também, que tanto o grupo caso, quanto o controle apresentaram melhora da assimetria facial após intervenção fonoaudiológica, pois a incompetência do movimento foi menor em todas as medidas, porém, o grupo caso apresentou maior redução da IM na medida canto externo do olho e comissura labial (CEO-
CL), localização dos músculos zigomáticos que auxiliam no movimento de sorriso aberto (Tabela 2).

Nos dois grupos, houve melhora na mímica facial após a intervenção, sinalizada pelo aumento do número de pacientes classificados com paralisia facial de graus I e II. Apenas no grupo controle houve diferença estatisticamente significativa nos resultados da disfunção moderada (grau III) pré e pósintervenção. No entanto, não se pode afirmar, com base nos dados da tabela, que o grupo controle obteve melhor resultado em relação ao grupo caso, pois os grupos não foram pareados pelo grau da PF (Tabela 3) e (Figura 2).

Embora no grupo controle a melhora tenha sido maior, percentualmente, não houve relevância estatística, indicando que os dois grupos obtiveram porcentagens semelhantes de melhora no grau de paralisia facial. (Tabela 4).

Ao analisar se a idade poderia ter influenciado nos resultados da incompetência do movimento após a intervenção, verificouse que não houve influência dessa variável nas medidas faciais (Tabela 5).

Tabela 1. Caracterização dos questionários por grupos e no total

\begin{tabular}{|c|c|c|c|c|c|c|c|c|}
\hline & & \multicolumn{2}{|c|}{ Total } & \multicolumn{2}{|c|}{ Caso } & \multicolumn{2}{|c|}{ Controle } & \multirow{2}{*}{$\begin{array}{l}\text { Valor } \\
\text { de } p^{*}\end{array}$} \\
\hline & & $\mathbf{n}$ & $\%$ & $\mathbf{n}$ & $\%$ & $n$ & $\%$ & \\
\hline \multirow[t]{2}{*}{ Grupo etário } & Adulto & 22 & 47,83 & 9 & 39,13 & 13 & 56,52 & 0,376 \\
\hline & Idoso & 24 & 52,17 & 14 & 60,87 & 10 & 43,48 & \\
\hline \multirow[t]{2}{*}{ Sexo } & Masculino & 28 & 60,87 & 10 & 43,48 & 18 & 78,26 & 0,033 \\
\hline & Feminino & 18 & 39,13 & 13 & 56,52 & 5 & 21,74 & \\
\hline \multirow[t]{2}{*}{ Tipo de AVC (HD) } & AVCi & 44 & 95,65 & 22 & 95,65 & 22 & 95,65 & 0,999 \\
\hline & $\begin{array}{l}\text { AVCi com transformação em } \\
\text { AVCh }\end{array}$ & 2 & 4,35 & 1 & 4,35 & 1 & 4,35 & \\
\hline \multirow{3}{*}{$\begin{array}{l}\text { Grau de comprometimento } \\
\text { neurológico (NIHHS) }\end{array}$} & Leve & 12 & 26,09 & 4 & 17,39 & 8 & 34,78 & 0,278 \\
\hline & Moderado & 25 & 54,35 & 15 & 65,22 & 10 & 43,48 & \\
\hline & Grave & 9 & 19,57 & 4 & 17,39 & 5 & 21,74 & \\
\hline \multirow[t]{3}{*}{$\begin{array}{l}\text { Medida de independência } \\
\text { funcional (pré-intervenção } \\
\text { fonoaudiológica) }\end{array}$} & $\begin{array}{l}\text { Dependência modificada com } \\
\text { assistência em até } 50 \% \text { na } \\
\text { tarefa }\end{array}$ & 27 & 58,70 & 15 & 65,22 & 12 & 52,17 & 0,707 \\
\hline & $\begin{array}{l}\text { Dependência modificada com } \\
\text { assistência em até } 25 \% \text { na } \\
\text { tarefa }\end{array}$ & 6 & 13,04 & 3 & 13,04 & 3 & 13,04 & \\
\hline & $\begin{array}{l}\text { Independência completa / } \\
\text { modificada pré-intervenção }\end{array}$ & 13 & 28,26 & 5 & 21,74 & 8 & 34,78 & \\
\hline \multirow[t]{2}{*}{ Lado da face acometido } & Direito & 17 & 36,96 & 10 & 43,48 & 7 & 30,43 & 0,542 \\
\hline & Esquerdo & 29 & 63,04 & 13 & 56,52 & 16 & 69,57 & \\
\hline \multirow[t]{2}{*}{ Tipo de paralisia facial } & Periférica & 3 & 6,52 & 2 & 8,70 & 1 & 4,35 & 0,999 \\
\hline & Central & 43 & 93,48 & 21 & 91,30 & 22 & 95,65 & \\
\hline \multirow{6}{*}{$\begin{array}{l}\text { Grau da paralisia } \\
\text { facial (pré-intervenção } \\
\text { fonoaudiológica) }\end{array}$} & Grau II - Disfunção leve & 9 & 19,57 & 3 & 13,04 & 6 & 26,09 & 0,600 \\
\hline & Grau III - Disfunção moderada & 18 & 39,13 & 10 & 43,48 & 8 & 34,78 & \\
\hline & $\begin{array}{l}\text { Grau IV - Disfunção moderada } \\
\text { grave }\end{array}$ & 7 & 15,22 & 5 & 21,74 & 2 & 8,70 & \\
\hline & Grau V - Disfunção grave & 10 & 21,74 & 4 & 17,39 & 6 & 26,09 & \\
\hline & Grau VI - Paralisia total & 2 & 4,35 & 1 & 4,35 & 1 & 4,35 & \\
\hline & & Mediana & $\begin{array}{l}\text { Média } \\
(\mathrm{DP} \pm)\end{array}$ & Mediana & $\begin{array}{l}\text { Média } \\
(\mathrm{DP} \pm)\end{array}$ & Mediana & $\begin{array}{l}\text { Média } \\
(\mathrm{DP} \pm)\end{array}$ & \\
\hline \multicolumn{2}{|l|}{ Idade (anos) } & 60,5 & $\begin{array}{c}62.4 \\
(\mathrm{DP} \pm 11,7)\end{array}$ & 65,00 & $\begin{array}{c}65.7 \\
(\mathrm{DP} \pm 10,6)\end{array}$ & 57,00 & $\begin{array}{c}59.1 \\
(\mathrm{DP} \pm 11.9)\end{array}$ & $0,057^{1}$ \\
\hline \multicolumn{2}{|l|}{ Tempo de intervenção (dias) } & 2,00 & $\begin{array}{c}2.96 \\
(\mathrm{DP} \pm 2,64)\end{array}$ & 2,00 & $\begin{array}{c}2.91 \\
(\mathrm{DP} \pm 2.48)\end{array}$ & 3,00 & $\begin{array}{c}3.00 \\
(\mathrm{DP} \pm 2.84)\end{array}$ & $0,477^{2}$ \\
\hline
\end{tabular}

Fonte: Unidade de AVC do Hospital Risoleta Tolentino Neves

*Teste Qui-Quadrado ou Exato de Fisher; ${ }^{1}$ Teste $t$ de Student para amostras independentes; ${ }^{2}$ Teste de Mann-Whitney

Legenda: $\mathrm{n}$ = número de sujeitos; $\mathrm{AVCi}$ = acidente vascular cerebral isquêmico; $\mathrm{AVCh}$ = acidente vascular cerebral hemorrágico; HD = high definition; $\mathrm{NIHHS}=$ National Institute of Health Stroke Scale; DP = desvio padrão 
Tabela 2. Comparação das medidas de incompetência do movimento da face no pré e pós-intervenção

\begin{tabular}{|c|c|c|c|c|c|c|c|c|c|c|c|}
\hline & \multirow{2}{*}{$\begin{array}{l}\text { Medidas da } \\
\text { face }(\mathbf{c m})\end{array}$} & \multicolumn{4}{|c|}{ Pré-intervenção } & \multicolumn{4}{|c|}{ Pós-intervenção } & \multirow{2}{*}{$\begin{array}{l}\text { Diferença } \\
\text { da média } \\
\text { (pré-pós) }\end{array}$} & \multirow{2}{*}{ Valor de $p^{*}$} \\
\hline & & Mín & Máx & Mediana & Média (DP) & Mín & Máx & Mediana & Média (DP) & & \\
\hline \multirow{4}{*}{$\begin{array}{c}\text { Grupo caso } \\
(n=23)\end{array}$} & IMT-CL & 0,00 & 22,90 & 11,80 & $11,76(5,75)$ & 0,00 & 15,80 & 6,20 & $6,13(3,88)$ & 5,63 & $<0,001$ \\
\hline & IM CIO-CL & 1,00 & 29,20 & 7,20 & $8,29(6.56)$ & 0,00 & 59,00 & 1,60 & $5,66(12,36)$ & 2,63 & 0,357 \\
\hline & IM CEO-CL & 1,00 & 28,30 & 10,80 & $11,70(7,57)$ & 0,00 & 18,40 & 3,00 & $3,71(4,10)$ & 8,00 & $<0,001$ \\
\hline & IM CIO-NA & 0,00 & 43,70 & 1,00 & $5,27(10,13)$ & 0,00 & 16,50 & 0,00 & $1,57(3,94)$ & 3,70 & 0,023 \\
\hline \multirow{4}{*}{$\begin{array}{c}\text { Grupo } \\
\text { controle } \\
(n=23)\end{array}$} & IM T-CL & 4,60 & 88,80 & 11,40 & $16,31(17,57)$ & $-4,80$ & 69,40 & 3,50 & $8,53(14,80)$ & 7,78 & 0,102 \\
\hline & IM CIO-CL & -1.20 & 22,90 & 7,60 & $8,42(6,14)$ & 0,00 & 20,90 & 3,50 & $4,73(5,07)$ & 3,70 & $<0,001$ \\
\hline & IM CEO-CL & 0,30 & 33,20 & 7,20 & $10,17(8,36)$ & $-5,40$ & 30,60 & 3,50 & $4,97(7,23)$ & 5,19 & $<0,001$ \\
\hline & IM CIO-NA & 0,00 & 30,90 & 5,10 & $7,94(8,49)$ & 0,00 & 27,50 & 2,60 & $4,11(5,90)$ & 3,83 & 0,012 \\
\hline
\end{tabular}

*Teste t de Student para amostras pareadas

Legenda: $\mathrm{cm}$ = centímetros; $\mathrm{n}$ = número de sujeitos; Mín = mínima; Máx = máxima; $\mathrm{DP}$ = desvio padrão; $\mathrm{IM}$ = incompetência do movimento; $\mathrm{T}-\mathrm{CL}=$ targus e comissura labial; $\mathrm{ClO}-\mathrm{CL}=$ canto interno do olho e comissura labial; $\mathrm{CEO}-\mathrm{CL}$ = canto externo do olho e comissura labial; $\mathrm{CIO}-\mathrm{NA}=$ canto interno do olho e ângulo nasal

Tabela 3. Grau de comprometimento da paralisia facial pré e pós-intervenção

\begin{tabular}{|c|c|c|c|c|c|c|}
\hline & Grau da paralisia facial & \multicolumn{2}{|c|}{ Pré-intervenção } & \multicolumn{2}{|c|}{ Pós-intervenção } & \multirow{2}{*}{ Valor de $p$} \\
\hline & (Escala de House \& Brackman) & $\mathbf{n}$ & $\%$ & $\mathbf{n}$ & $\%$ & \\
\hline \multirow{6}{*}{$\begin{array}{c}\text { Grupo caso } \\
(n=23)\end{array}$} & Grau I- Normal & 0 & 0,00 & 2 & 8,70 & 0.489 \\
\hline & Grau II - Disfunção leve & 3 & 13,04 & 7 & 30,43 & 0.153 \\
\hline & Grau III - Disfunção moderada & 10 & 43,48 & 7 & 30,43 & 0.359 \\
\hline & Grau IV - Disfunção moderada grave & 5 & 21,74 & 4 & 17,39 & 0.999 \\
\hline & Grau V - Disfunção grave & 4 & 17,39 & 3 & 13,04 & 0.999 \\
\hline & Grau VI - Paralisia total & 1 & 4,35 & 0 & 0,00 & 0.999 \\
\hline \multirow{6}{*}{$\begin{array}{l}\text { Grupo controle } \\
\qquad(n=23)\end{array}$} & Grau I - Normal & 0 & 0,00 & 4 & 17,39 & 0,109 \\
\hline & Grau II - Disfunção leve & 6 & 26,09 & 9 & 39,13 & 0,345 \\
\hline & Grau III - Disfunção moderada & 8 & 34,78 & 2 & 8,70 & 0,032 \\
\hline & Grau IV - Disfunção moderada grave & 2 & 8,70 & 3 & 13,04 & 0,999 \\
\hline & Grau V - Disfunção grave & 6 & 26,09 & 5 & 21,74 & 0,730 \\
\hline & Grau VI - Paralisia total & 1 & 4,35 & 0 & 0,00 & 0,999 \\
\hline
\end{tabular}

*Teste Qui-Quadrado ou Exato de Fisher

Legenda: $\mathrm{n}=$ número de sujeitos

Tabela 4. Análise da melhora do grau da paralisia facial após a intervenção fonoaudiológica

\begin{tabular}{|c|c|c|c|c|c|}
\hline \multirow{2}{*}{$\begin{array}{l}\text { Grau da Paralisia } \\
\text { Facial pós- } \\
\text { intervenção }\end{array}$} & \multicolumn{2}{|c|}{ Grupo Caso } & \multicolumn{2}{|c|}{ Grupo Controle } & \multirow{2}{*}{ Valor de $p^{\star \star}$} \\
\hline & $\mathbf{n}$ & $\%$ & $\mathrm{n}$ & $\%$ & \\
\hline Melhorou & 12 & 52,17 & 15 & 65,22 & \\
\hline Piorou & 0 & 0,00 & 0 & 0,00 & 0,550 \\
\hline Manteve & 11 & 47,83 & 8 & 34,78 & \\
\hline Valor $p^{*}$ & \multicolumn{2}{|c|}{$<0,001$} & \multicolumn{2}{|c|}{$<0,001$} & \\
\hline
\end{tabular}

*Teste de Wilcoxon; **Teste Qui-Quadrado

Legenda: $\mathrm{n}=$ número de sujeitos

Tabela 5. Associação entre a incompetência do movimento facial e a idade

\begin{tabular}{|c|c|c|c|c|c|c|c|c|}
\hline \multirow{3}{*}{$\begin{array}{c}\text { Variável dependente } \\
\text { - Idade }\end{array}$} & \multicolumn{4}{|c|}{ Grupo caso $(n=12)$} & \multicolumn{4}{|c|}{ Grupo controle $(n=15)$} \\
\hline & \multirow{2}{*}{ OR } & \multirow{2}{*}{ Valor de $p^{*}$} & \multicolumn{2}{|c|}{$\begin{array}{c}\text { Intervalo de Confiança } \\
95 \% \text { para OR } \\
\end{array}$} & \multirow{2}{*}{ OR } & \multirow{2}{*}{ Valor de $\mathrm{p}^{*}$} & \multicolumn{2}{|c|}{$\begin{array}{c}\text { Intervalo de Confiança } \\
95 \% \text { para OR }\end{array}$} \\
\hline & & & $\begin{array}{l}\text { Limite } \\
\text { inferior }\end{array}$ & $\begin{array}{l}\text { Limite } \\
\text { superior }\end{array}$ & & & $\begin{array}{l}\text { Limite } \\
\text { inferior }\end{array}$ & $\begin{array}{l}\text { Limite } \\
\text { superior }\end{array}$ \\
\hline IM T-CL & $-0,224$ & 0,647 & $-1,281$ & 0,832 & 0,236 & 0,729 & $-1,243$ & 1,716 \\
\hline IM CIO-CL & 0,456 & 0,172 & $-0,235$ & 1,147 & 0,004 & 0,983 & $-0,366$ & 0,373 \\
\hline IM CEO-CL & 0,501 & 0,140 & $-0,196$ & 1,197 & $-0,176$ & 0,750 & $-1,376$ & 1,023 \\
\hline IM CIO-NA & 0,330 & 0,132 & $-0,118$ & 0,779 & 1,583 & 0,076 & $-0,203$ & 3,369 \\
\hline
\end{tabular}

\footnotetext{
${ }^{*}$ Regressão linear simples
}

Legenda: $\mathrm{n}=$ número de sujeitos; $\mathrm{OR}=$ odds ratio; $\mathrm{IM}$ = incompetência do movimento; $\mathrm{T}-\mathrm{CL}=$ targus e comissura labial; $\mathrm{ClO}-\mathrm{CL}=$ canto interno do olho e comissura labial; $\mathrm{CEO}-\mathrm{CL}$ = canto externo do olho e comissura labial; CIO-NA = canto interno do olho e ângulo nasal 


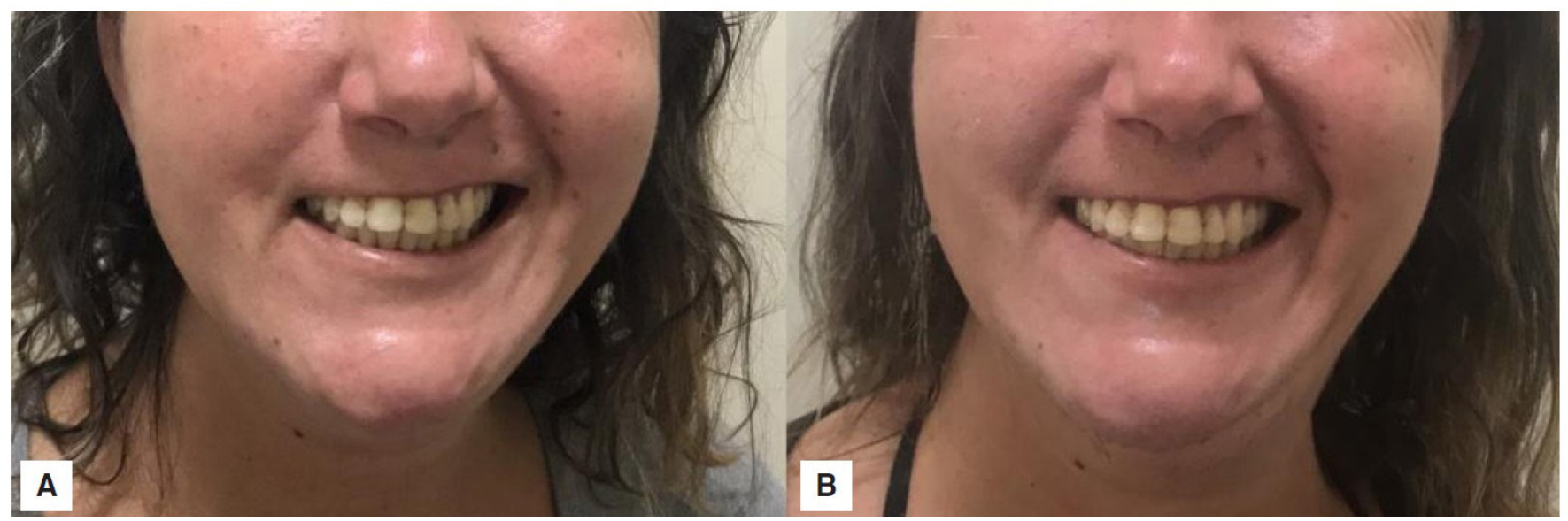

Figura 2. (A) Antes da aplicação da fita de Kinésio; (B) Duas semanas após o início da utilização da fita de Kinésio

\section{DISCUSSÃO}

Este é o primeiro estudo que investigou a terapia miofuncional orofacial exclusiva e associada ao uso da KT na reabilitação da paralisia facial pós-AVC na fase aguda e não houve diferença entre os grupos de pacientes.

A atuação fonoaudiológica na reabilitação da paralisia facial não abrange somente a redução da assimetria, mas também atua sobre a função que essa musculatura exerce no sistema estomatognático $^{(20)}$. Na Fonoaudiologia, o uso da bandagem de KT é indicado para favorecer o trabalho muscular, gerando benefícios diretos sobre as funções orofaciais ${ }^{(16-19)}$. A KT não é um tratamento, mas um recurso adicional que complementa as técnicas utilizadas, devido a sua ação contínua, potencializando os resultados da terapia tradicional e auxiliando na estimulação da neuroplasticidade ${ }^{(16)}$.

Os estudos que investigaram os efeitos do uso da KT na reabilitação dos pacientes com disfunções neurológicas mostraram que os principais benefícios foram a melhora da propriocepção e a normalização da função muscular no local onde a bandagem foi aplicada ${ }^{(8-14)}$. As modificações no sistema nervoso central (SNC) são realizadas por estímulos sensoriais, motores, experiências e aprendizados. Sendo assim, a bandagem elástica atua como fonte de estímulo sensorial, buscando recuperar ou melhorar a performance do movimento. $\mathrm{O}$ estímulo somatossensorial pode ativar vários receptores, e cabe ao SNC interpretar a atividade deles e usá-los para gerar novas percepções ${ }^{13}$. Portanto, o uso da KT ainda na fase aguda pós-AVC é um recurso adicional à terapia miofuncional orofacial, sendo um estímulo externo que pode propiciar a neuroplasticidade do nervo facial, promovendo a regeneração axonal.

Em relação ao percentual de redução do grau da paralisia facial, a maioria dos pacientes que apresentou melhora foi do grupo controle. Isso se deve ao maior número de indivíduos com grau mais leve da PF na pré-intervenção e, consequentemente, a melhora da simetria facial é alcançada em menor tempo.

Houve melhora significativa da incompetência do movimento (IM) pós-intervenção nos grupos caso e controle, achado que reforça o que a literatura já preconiza sobre a eficácia da terapia miofuncional orofacial na reabilitação desses pacientes ${ }^{(16-19)}$.

São vários os estudos que descrevem os benefícios da reabilitação miofuncional orofacial nas PFs, uma vez que é possível promover a redução da assimetria facial, melhora da alimentação, da fala e dos problemas emocionais, diminuindo os impactos negativos na qualidade de vida desses indivíduos ${ }^{(26-29)}$.

A intervenção precoce é um indicativo de bom prognóstico na reabilitação das $\mathrm{PFs}^{(27-28)}$, colaborando para o retorno da mobilidade e funções da musculatura orofacial e reduzindo, assim, a atrofia muscular e surgimento de sincinesias e contraturas $^{(21)}$. Nesse aspecto, a atuação fonoaudiológica neste estudo se beneficiou pela possibilidade de diagnóstico clínico precoce da $\mathrm{PF}$ e o início rápido da reabilitação, ainda na fase aguda do AVC.

Neste estudo, não foi observada diferença significativa entre a utilização, ou não, da KT na reabilitação dos pacientes com PF pós-AVC. Esse fator pode ser justificado pelo tempo curto de acompanhamento dos pacientes, sendo que o tempo médio para reabilitação das paralisias faciais é de três a 12 meses, de acordo com o balizador de tempo de tratamento em Fonoaudiologia, e o tempo de internação na unidade de AVC, em torno de 15 dias $^{(30)}$.

Vale mencionar que os resultados deste estudo sugeriram que a KT pode ser um recurso adicional utilizado para promover retorno à mímica facial após AVC. No grupo em que a KT foi aplicada nos músculos zigomáticos maior e menor, a média da incompetência do movimento pré e pós-intervenção apresentou maior diferença, indicando boa resposta terapêutica, embora sem relevância estatística. Esse resultado deve ser melhor investigado em estudos futuros e com acompanhamento por mais tempo dos pacientes, a fim de verificar se a KT associada à terapia miofuncional orofacial melhora, de fato, a incompetência do movimento, quando comparada ao uso das estratégias tradicionais da reabilitação fonoaudiológica.

É importante ressaltar que a aplicação da KT na face se difere da aplicação em outros músculos do corpo humano ${ }^{(8-13)}$, pois a musculatura orofacial é composta por músculos delgados cutâneos que, de um modo geral, são menores que os demais músculos corporais e mais próximos uns dos outros, formando um grupo muscular complexo de ser trabalhado ${ }^{(26-29)}$. Então, pergunta-se: no caso da paralisia facial, seria mais benéfico bandar os músculos isoladamente ou bandar o grupo muscular responsável por um determinado movimento da mímica facial? Teria o modo de aplicação da bandagem interferido nos resultados encontrados nesta amostra? Para responder a essas indagações, 
são necessários trabalhos que abordem a biomecânica da aplicação da KT na musculatura orofacial.

Não foram encontrados, na literatura, trabalhos que tenham utilizado a KT na reabilitação das PFs, assim como é reduzido o número de publicações que abordam as PFC, quando comparado a publicações sobre PFP. Optou-se por iniciar os estudos de bandagem em pacientes com PF pós-AVC na fase aguda, pois seria mais confiável o controle da realização dos exercícios propostos nos pacientes internados, uma vez que a fonoaudióloga poderia executar diariamente os exercícios à beira leito, o que não seria possível com o paciente em domicílio, situação em que a profissional teria que confiar no relato do paciente sobre a realização das tarefas propostas. Além disso, ressalta-se a importância de investigar, nessa população, técnicas que promovam a neuroplasticidade e a intervenção precoce, que é um fator de bom prognóstico para reduzir o índice de morbidade e incapacidade.

Este estudo é um importante alerta sobre a necessidade de pesquisas para a padronização desse recurso na reabilitação das paralisias faciais. Vale destacar que o uso do protocolo de medição da face ${ }^{(26)}$ permitiu quantificar a incompetência do movimento e comparar, na pré e pós-intervenção a ação dos músculos que receberam a $\mathrm{KT}$, tornando os resultados confiáveis e permitindo comparações objetivas com estudos futuros. Possibilidade de novos estudos com maior número de pacientes e tempo de tratamento, assim como de outros protocolos de avaliação, como a eletromiografia, complementando esses achados, é relevante e necessária.

O fonoaudiólogo tem recorrido a diferentes recursos tecnológicos e esses precisam ser mais estudados ${ }^{(16)}$, a fim de que as evidências científicas sejam balizadoras desses novos métodos terapêuticos.

\section{CONCLUSÃO}

Não houve diferença estatisticamente significativa entre a terapia miofuncional orofacial exclusiva ou associada ao uso da fita de Kinesio Taping na reabilitação das paralisias faciais pós-acidente vascular cerebral isquêmico na fase aguda. Os dois métodos mostraram ser eficazes no tratamento do comprometimento da mímica facial e, portanto, a bandagem elástica pode ser utilizada associada à terapia miofuncional orofacial nas paralisias faciais pós-avc.

\section{AGRADECIMENTOS}

Aos pacientes, preceptores e colegas do Hospital Risoleta Tolentino Neves.

\section{REFERÊNCIAS}

1. Calais LL, Gomez MVSG, Bento RF, Comerlatti LR. Mime functional evaluation in facial paralysis following a stroke. Pro Fono. 2005;17(2):213-22. http://dx.doi.org/10.1590/S0104-56872005000200010. PMid:16909531.

2. Pimenta E, Costa A, Buke MJ. Strategies for Recovering facial expression after central facial paresis. RIASE. 2019;5(1):1691-706. http://dx.doi.org/10.24902/r.riase.2019.5(1).1691.
3. Konecny P, Elfmark M, Horak S, Pastucha D, Krobot A, Urbanek $\mathrm{K}$, et al. Central facial paresis and its impact on mimicry, psyche and quality of life in patients after stroke. Biomed Pap Med Fac Univ Palacky Olomouc Czech Repub. 2014;158(1):133-7. http://dx.doi. org/10.5507/bp.2013.014. PMid:23558454.

4. Pereira, KJ, Rios MJBL, Silva DR, Silva MRDCD, Ribeiro JM, Barbosa IS. Physiotherapy in facial paralysis. ReonFacema. 2018;(4):1339-43.

5. Silva MFF, Brito AFD, Campos MF, Cunha MC. Multidisciplinary care of peripheral facial palsy: clinical case study. Distúrb Comum. 2015;27(2):364-8.

6. Souza IF, Dias ANM, Fontes FP, Melo LP. Physical therapy methods used in the treatment of peripheral facial paralysis: a review. Rev Bras Ciênc Saúde. 2015;19(4):315-20.

7. Guimarães SS, Ferreira DM, Silva AM, Silva AT, Kosour C, Reis LM Effect of Taping with or without FES and vibration in dorsiflexion and weight bearing after stroke. Rev Neurocienc. 2015;23(3):383-9. http://dx.doi.org/10.4181/RNC.2015.23.03.1026.06p.

8. Henriques LFDM, Wnderley D, Lima DLP, Filho AGM, Oliveira DAD. Kinesio taping on the balance gain in patients after stroke. Braz J Hea Ver. 2019;2(1):290-303.

9. Pereira AA Jr, Butzke J, Persuhn JJ. Kinesio Taping $®$ in association with kinesiotheraphy on gait correction of after stroke. Rev Pesqui Fisioter. 2016;(1):73-82.

10. Magalhães HCCDG, Menezes KKPD, Avelino PR. Efeitos do uso do Kinesio ${ }^{\circledR}$ Taping na marcha de indivíduos pós-acidente vascular encefálico: uma revisão sistemática com metanálise. Fisioter Pesqui. 2017;24(2):218-28. http://dx.doi.org/10.1590/1809-2950/17387024022017.

11. Hu Y, Zhong D, Xiao Q, Chen Q, Juan L, Jin R. Kinesio Taping for balance function after stroke: a systematic review and meta-analysis. CAM. 2019;2019:8470235.

12. Morini N Jr, Santos SMS, Caramello AG, Maidana MG, Aquiahuatl CM, Silva APD, et al. Bandagem terapêutica: conceito de estimulação tegumentar. 2a ed. São Paulo: Editora Roca; 2016.

13. Araújo GJS, Rodrigo AS, Cavalcante MLC, Moraes MRB. A aplicabilidade do recurso Kinesio Taping ${ }^{\circledR}$ nas lesões desportivas: uma revisão de literatura. Rev Pesqui Fisioter. 2014;4(3):189-96.

14. Lemos TV, Pereira KC, Protássio CC, Lucas LB, Matheus JPC. The effect of Kinesio Taping on handgrip strength. J Phys Ther Sci. 2015;27(3):567-70. http://dx.doi.org/10.1589/jpts.27.567. PMid:25931682.

15. Vithoulka I, Beneka A, Malliou P, Aggelousisb N, Karatsolisa K, Diamantopoulos K. The effects of kinesio-taping on quadriceps strength during isokinetic exercise in healthy non athelete women. Isokinet Exerc Sci. 2010;42:11-6.

16. Silva AP, Escamez NES, Morini N Jr, Silva MAA. Therapy Taping ${ }^{\circledR}$ Method: therapeutic taping as a therapeutic resource in the Speech Language clinical practices. Distúrb Comun. 2014;26(4):805-8.

17. Sordi C, Araújo BL, Cardoso LVD, Correia LAV, Oliveira GM, Silva SSS, César CPHAR. Elastic bandage as a therapeutic resource for the control of sialorrhea: an analysis of its efficacy. Distúrb Comum. 2017;29(4):663-72.

18. Hernandes NCJ, Ribeiro LL, Gomes CF, Silva AP, Dias VF. Speech therapy in temporomandibular dysfunction in two cases: comparative analysis of the effect of traditional therapy and the use of the therapeutic bandage associated. Distúrb Comun. 2017;29(2):251-60. http://dx.doi. org/10.23925/2176-2724.2017v29i2p251-261.

19. Mezzedimi C, Livi W, Spinosi MC. Kinesio Taping in Dysphonic Patients. J Voice. 2017;31(5):589-93. http://dx.doi.org/10.1016/j. jvoice.2017.01.012. PMid:28237552. 
20. Mota DVN, Silva LVC. Use of functional bandages in patients with stroke. Fisioter Mov. 2014;27(3):329-36. http://dx.doi.org/10.1590/01035150.027.003.AO03.

21. Ramos RDL. Adaptação cultural e validação do "The Language Screening Test- LAST" versões a e b para o português do Brasil [dissertação]. Campinas: Faculdade Ciências Médicas da Universidade Estadual de Campinas - UNICAMP; 2013.

22. Cincura C, Pontes-Neto OM, Neville IS, Mendes HF, Menezes DF, Mariano DC, et al. Validation of the national institutes of health stroke scale modified Rankin Scale and Barthel index in Brazil: the role of cultural adaptation and structured interviewing. Cerebrovasc Dis. 2009;27(2):119-22. http://dx.doi.org/10.1159/000177918. PMid:19039215.

23. Riberto M, Miyazaki MH, Jucá SSH, Sakamoto H, Pinto PPN, Battistella LR. Validation of the Brazilian version of functional independence measure. Acta Fisiatr. 2004;11(2):72-6. http://dx.doi. org/10.5935/0104-7795.20040003.

24. House JW, Brackmann DE. Facial nerve grading system. Otolaryngol Head Neck Surg. 1985;93(2):146-47. http://dx.doi. org/10.1177/019459988509300202. PMid:3921901.

25. Fonseca KMO, Mourão A, Motta AR, Vicente LCC. Scales of degree of facial paralysis: analysis of agreement. Braz J Otorhinolaryngo.
2015;81(3):288-93. http://dx.doi.org/10.1016/j.bjorl.2014.04.005. PMid:25497850.

26. Quintal M, Tessitore A, Paschoal JR, Pfeilsticker LN. Facial paralysis quantification by digital paquimeter. Rev CEFAC. 2013;15(3):592-8.

27. Miranda VHM, Scarpel VR, Torres ACM, Agra IMG. Effectiveness of speech therapy in patients with facial paralysis after parotidectomy. Rev CEFAC. 2015;17(3):984-95. http://dx.doi.org/10.1590/1982-021620157314.

28. Romão AM, Cabral C, Magni C. Early speech therapy intervention in a patient with facial paralysis after otomastoiditis. Rev CEFAC. 2015;17(3):996-1003.

29. Tessitore A, Pfelsticker LN, Paschoal JR. Neurophysiologic aspects of facial musculature aiming at rehabilitation of Facial Paralysis. Rev CEFAC. 2008;10(1):68-75. http://dx.doi.org/10.1590/S151618462008000100010.

30. Mourão AM, Vicente LCC, Chaves TS, Sant'Anna RV, Meira FC, Xavier RMB, et al. Profile of patients with a diagnosis of stroke attended at a hospital in Minas Gerais accredited in the care line. Rev Bras Neurol. 2017;53(4):12-6. 